\title{
Partner Selection, Power Dynamics, and Mutual Care Giving in Long-Term Self-Defined BDSM Couples
}

\author{
Bert Cutler ${ }^{1}$ \\ Tempe, AZ (USA) \\ bertcutler@ingratitude.net \\ Ellen M. Lee \\ Coe College (USA) \\ Nadine Cutler \\ Tempe, AZ (USA) \\ Brad J. Sagarin \\ Northern Illinois University (USA)
}

\begin{abstract}
Historically, writers from within and outside the BDSM/Leather community have argued that long-term BDSM relationships are likely to fail due to the incompatibility between satisfying SM and romantic affection (Townsend, 1972) and the unlikelihood of finding a perfect match of fetishes and interests (Money, 1986). The present study tested these arguments using in-depth interviews with 33 individuals in 17 long-term BDSM relationships. Qualitative and quantitative methods were used to understand the dynamics of the relationships including the ways the partners met, formed their relationship, negotiated the scope of their power exchange, and navigated the challenges of an unconventional relationship style. Compatibility regarding dominance and submission appeared more important than compatibility regarding sadism and masochism or specific BDSM activities. Power exchange appears to serve purposes beyond sexual satisfaction, including facilitating partner bonding and providing a sense of security. Key relationship values that emerged across couples include a commitment to communication and transparency, a high level of trust, a focus on the partner's happiness, and the co-construction of a reality that satisfies the needs of both partners. The use of rituals and protocol in difficult times returned the partners to a state of connection to each other, and this re-established bond helped the partners resolve the present upset. The use of deeper protocol when issues arise is contrary to prevailing community rhetoric that advises dropping out of role to solve issues. Thus, rituals give these individuals a different, possibly safer, way to interact so that conflict doesn't damage their relationships. Results demonstrate that long-term BDSM relationships exist and can be highly functional.
\end{abstract}

\footnotetext{
${ }^{1}$ Author Note: We thank the respondents of this study who trusted enough to reveal intimate views into the details of their lives and relationships. The data for this paper originated from the first author's doctoral dissertation, which was completed at the Institute for the Advanced Study of Human Sexuality. The first author gives special thanks to Dr. Clark Taylor who provided academic guidance from the beginning and to the Science of BDSM Research Team at Northern Illinois University for their feedback.
} 
In 2015, Joyal, Cossette, and Lapierre asked 1516 Canadian adults to rate the intensity of their interest in 55 sexual fantasies. At least $62 \%$ of respondents reported the presence of at least one fantasy involving BDSM (Bondage/Discipline, Dominance/Submission, Sadism/Masochism). In 2017, Holvoet and colleagues surveyed 1027 Belgian adults regarding their interest in 54 BDSM activities. A total of $68.8 \%$ of respondents reported fantasizing about or participating in at least one activity.

Given the prevalence of interest in BDSM, it is not surprising that researchers have focused increasing attention on the subject, offering insights into the demographics and personalities of BDSM practitioners (e.g., Hébert \& Weaver, 2014; Wismeijer \& van Assen, 2013), the taxonomies and effects of BDSM activities (e.g., Alison et al., 2001; Sagarin et al., 2009), and the functions and benefits of BDSM communities (e.g., Graham et al., 2015) (see Brown et al., 2020; De Neef et al., 2019, for reviews). However, one particular topic has received relatively little attention from researchers: BDSM relationships. In their review of research on relationship diversity, Hammack, Frost, and Hughes (2019) highlight this imbalance:

We note that overt discussion of kink relationships is extraordinarily rare in the literature. Rather, most research emphasizes kinky "practices" or "scenes" in a way that implicitly delegitimizes the connection among partners as ephemeral or linked mainly to individual pleasure. We call for increased research on relationships that are explicitly defined as kinky. (p. 576)

This imbalance has both theoretical and practical consequences. At a theoretical level, social scientists have only a rudimentary understanding of a relationship form that differs in fundamental ways from normative romantic and sexual relationships. At a practical level, therapists and clinicians have limited evidence to guide their practice when working with clients in BDSM relationships (but see Shahbaz \& Chirinos, 2016, for a research-informed guide for therapists serving kink clients).

The present study joins a small set of published studies in beginning to rectify this imbalance. To set a context for the present study, we begin by reviewing past research on BDSM relationships including the four features that Hammack et al. (2019) identify as characterizing such relationships. We then define relevant terminology regarding BDSM practitioners, activities, and relationships. Thereafter, we describe the goals and design of the current study and present the methodology and results.

\section{Past Research on BDSM Relationships}

A subset of BDSM practitioners identify as being in, or wanting, a "power exchange" relationship, in which one partner has authority over the other in daily living, sometimes on a " $24 / 7$ " basis. One of the first academic inquiries into power exchange relationships was Dancer, Kleinplatz, and Moser's (2006) “24/7 SM Slavery.” Dancer et al. (2006) surveyed 146 slaveidentified individuals. The results revealed relationships in which the power exchange was pervasive and all-encompassing, with a high degree of ongoing ritual and protocol. Slaves were treated as partners in the relationship, and structures were in place in order to safeguard the physical and psychological safety of the slave. The vast majority $(88 \%)$ of participants reported 
being satisfied or completely satisfied with their relationship. Summarizing their findings, Dancer et al. (2006) explain that

There is no reason to believe that these 'slaves' need saving or freeing. There is no indication that they are more likely to be abused than in other relationships. It would appear that some of the shock from disclosing participation in this type of relationship relates to the terms used to describe the relationship, rather than the actual behavior within the relationship. (p. 99)

Butts (2007) presents a case study that explores body ownership in the context of the relationships between a Mistress and her slaves. Highlighting the pervasive nature of power dynamics in these relationships, Butts explains:

It is significant that I do not use the phrase "sexual behavior patterns" in this statement, as Mistress Linda's choice to go 24/7 with her M/s identification means the dynamic she establishes with her slaves is not limited to the times during which they are engaged in sex play. This highlights the fact that $\mathrm{M} / \mathrm{s}$ relationships are, in fact, relationships, and as such, they are not as far removed from more typical forms of intimate partnerships as one might suspect. (p. 71, italics in the original)

Consistent with this perspective, Bauer (2019) highlights the ways that studying BDSM relationships can deepen our understanding of relationships in general:

BDSM relationships therefore can serve as a starting point for theorizing intimacy from a perspective that focuses on issues of power and consent rather than notions of (an illusionary) equality and romantic love. (p. 145)

Rogak and Conner (2018) measured relationship satisfaction in a sample of 163 BDSM practitioners who reported being married or in a committed relationship. BDSM practitioners reported relationship satisfaction scores comparable to scores from a prior sample of nondistressed individuals (Crane et al., 2000) and substantially higher than scores from a prior sample of distressed individuals (Crane et al.), supporting the perspective that BDSM relationships can represent a healthy and satisfying relationship form.

BDSM community authors have also explored the dynamics of power exchange relationships. Townsend (1972) had a pessimistic view of such relationships, reporting few longlasting BDSM relationships and suggesting that Leathermen were best off selecting nonLeathermen for their primary partner and getting their BDSM needs met outside of that relationship. Baldwin (1993), in contrast, referenced long-lasting, working BDSM relationships within the gay leather community of Los Angeles. Baldwin's writings include numerous anecdotes about couples whose relationship roles and BDSM roles were fluid, reflecting the needs of the individuals and their circumstances. Other BDSM community writers have also discussed working relationships (Bean, 1994; Brame et al., 1993; Califa, 1993; Campbell, 2000), with some offering practical advice about negotiations and protocols. 
Hammack et al. (2019) offer a useful framework for understanding BDSM relationships:

Our review highlights four common features or experiences of kink relationships. First, such relationships are typically characterized by recreational power exchange. Second, they are consensual and collaboratively scripted. Third, they are typically embedded in a larger community of shared meaning and practices. Finally, because such relationships may be stigmatized, individuals may experience minority stress related to issues of concealment, disclosure, stigma, and internalized kinkphobia. (p. 576).

In the Discussion, we consider the implications of the present results for Hammack et al.'s framework.

\section{BDSM Terminology and Roles}

As an umbrella term, BDSM encompasses a wide range of activities and relationship styles (De Neef et al., 2019). Many definitions of BDSM have been presented within the academic literature as well as the community literature. One such example is Jay Wiseman's (1998) definition in SM 101:

The knowing use of psychological dominance and submission, and/or physical bondage, and/or pain, and/or related practices in a safe, legal, consensual manner in order for the participants to experience erotic arousal and/or personal growth. (p. 10)

Some common themes across these definitions include consent and a recognition that people may practice these behaviors for a multitude of reasons, such as eroticism, spirituality, catharsis, skill development, personal identity, and fun.

BDSM practitioners use a variety of terms to refer to their roles. For example, a person may refer to themselves as a Top or a Dominant (or a Master, Mistress, Handler, Daddy, etc.). Likewise, a person may refer to themselves as a bottom or a submissive (or a slave, pet, little, etc.). ${ }^{2}$ Although these terms are sometimes used interchangeably, there are important distinctions between these terms (and between different people's use of the terms). Below, we offer brief definitions of four terms relevant for the present study: Top, bottom, Dominant, and submissive:

"Top" and "bottom" are physical terms describing who is doing an act and who is receiving. For example, the Top is the partner using the flogger, or doing the tying, while the bottom is the one being flogged or being tied up. The person who is topping and the person who is bottoming can be determined by looking across the room at the activities occurring. Those who enjoy both topping and bottoming (usually not at the same time) may identify as a Switch.

"Dominant" and "submissive" are psychological terms. The Dominant is directing the action while the submissive is carrying out the directions. For example, the Dominant might direct the submissive to get them a cup of coffee. Likewise, the Dominant might direct the

\footnotetext{
${ }^{2}$ This paper adopts the capitalization convention of some BDSM communities in which the roles of Top, Dominant, etc. are capitalized, and the roles of bottom, submissive, etc. are lowercased.
} 
submissive to flog the Dominant, in which case the Dominant is bottoming and the submissive is topping.

\section{Power Exchange Relationships}

A Power Exchange relationship is a standing agreement to exchange power between the submissive and the Dominant, empowering the Dominant to provide orders and take responsibility for the results of those orders. A healthy Power Exchange relationship empowers the submissive and is not about the submissive becoming powerless. Personal power, in this context, refers to the ability to assume personal agency in one's life. Individuals in Power Exchange relationships negotiate the manifestations of personal power. For example, a submissive may have personal power with respect to their own healthcare but has consented to the Dominant deciding what clothes the submissive will wear.

The consensual, mutually desired exchange of power in Power Exchange relationships stands in contrast to the ways that power often manifests in conventional romantic relationships. Lennon, Stewart, and Ledermann (2012) define power as "the feeling derived from the ability to dominate, or control, the behavior, affect, and cognitions of another person" (p. 97). Relationship researchers have identified that power, and the subsequent vying for power, is a strong, pervasive feature of romantic dynamics (Dunbar, 2004; Lennon et al., 2012). Power is often not shared completely equally between partners (Amaro, 1995; Lennon et al., 2012), even though "contemporary couples increasingly express preferences for egalitarian unions" (Carlson, Miller, Sassler, \& Hanson, 2014, p. 975). Power may be distributed within a relationship based on skill, experience, personality, or intensity of preference. For example, one partner deciding which restaurant to eat at could be the result of that person's greater knowledge of local cuisine or because that person enjoys being in control. The way that power is negotiated within relationships can be constructive and progressive or destructive and relationship threatening (within long-term relationships it might well be both).

Individuals in Power Exchange relationships use a range of terms to describe their relationships including Dominant and submissive, Master and slave, Daddy and (adult) girl, Trainer and puppy, Trainer and pony, and many more. Lee, Erickson, and Sagarin (2018) surveyed 135 people in power exchange relationships. Participants identified their relationship styles using over 20 unique labels, including Magister and acolyte, Master alpha and slave beta, Sir and girl, Momma Bear and boi, and Commander and executive officer. BDSM community author Raven Kaldera's (2014) edited volume on different Master/slave styles entitled Paradigms of Power highlights how even within one subtype of power exchange relationships$\mathrm{M} / \mathrm{s}$ - there is great variety in intent, practice, and perceived benefits. The specific label people use for their relationship style seems to be driven strongly by the relationship partners: People adopt labels that they can feel comfortable with, and that they believe accurately and uniquely reflect their relationship dynamics.

\section{Current Study}

The current study consisted of interviews with 33 individuals in long-term BDSM relationships. The study used qualitative and quantitative methods to understand the dynamics of 
the relationships including how the partners met, formed their relationship, negotiated the scope of their power exchange, and navigated the challenges of an unconventional relationship style.

Qualitative methods were used to identify themes within each area of inquiry. Shortly after the interviews were concluded, the first author identified common themes that emerged across participants in the areas of partner selection, relationship dynamics, and power dynamics. This process began with the first author reflecting on his memory of the interviews followed by a review of the audiotaped interviews and the written transcripts produced by the third author. During this review, the first author began to identify sub-themes within each theme. For example, the sub-themes of power domains, power fluidity, and role switching emerged within the theme of power dynamics. Some of the sub-themes were anticipated in constructing the interview questions. For example, participants were asked specifically to describe the domains of power held by each partner within their relationship. Other sub-themes were not anticipated. For example, the sub-theme of atonement emerged from the interview question on punishment. Quantitative methods were used to statistically analyze how compatible the couples were in terms of dominance/submission, sadism/masochism, and topping/bottoming various BDSM activities. Details on the coding and analysis are presented in the Results section.

The interviews were collected May 2001 through July, 2002. Given the age of the data, it is reasonable to ask whether the data are still relevant. We believe they are. Although terminology has changed since 2002, the specific dynamics of power exchange relationships are likely still functioning in the same way. People are still dealing with the same issues of how to create a healthy, functional relationship today as they were in the early $2000 \mathrm{~s}$. We see three specific issues stemming from the age of the data: (a) The data preceded the publication of Fifty Shades of Grey (James, 2012). Although Fifty Shades of Grey is largely derided by the BDSM community as non-consensual, inaccurate, and misleading (Kimble, 2017; Kravitz, 2018; Smith, 2015), the book and movie have provided arguably the most well-known fictional representation of BDSM. Thus, power exchange couples today have (for good or for bad) Fifty Shades as an exemplar. (b) Social media currently provides BDSM practitioners a high level of connectivity to other practitioners. Couples in the study were members of a BDSM organization, but the pool of peer practitioners was substantially smaller than it might be today. (c) Terminology used to identify roles within a power exchange relationship have changed over the years. In the early 2000s timeframe, the term Master/slave (M/s) was not in common use within the community from which the sample was drawn. Today some of the respondents would likely use the term. We revisit the question of the relevance of the data in the Discussion section, particularly within the context of their implications for Hammack et al.'s (2019) proposed framework.

\section{Method}

\section{Participants}

The 33 participants consisted of 17 heterosexual couples from 16 households. One household consisted of one man with two women; the BDSM relationships were between the man and separately with each of the two women, a wife and a lover. The women did not consider that they had a BDSM relationship with each other. Using the respondents' initial self-labels, the 
couples in this study all fell into two classifications, Dominant/submissive (15) and Switch (2), and those labels are used to identify the qualitative quotes throughout this paper.

Seven couples were married. Four couples were engaged to be married at the time of their interviews and six had no marriage plans. All four engaged couples subsequently got married. The three couples with no marriage plans reported they had a life commitment. One couple reported that the collar ${ }^{3}$ is considered stronger than a wedding band.

The mean age of the men was 48 (range: 35 to 70); the mean age of the women was 40 (range: 20 to 53). Thirteen couples were male dominant. Two couples were female dominant. Two couples were switches, and for both switch couples, the male identified as a dominantleaning switch and the female identified as a submissive-leaning switch. Within the household with one man and two women, one relationship was male dominant, the other was switch.

\section{Procedure}

\section{Sample Selection}

The respondents in this study were 17 heterosexual couples in self-defined BDSM relationships who engaged in sadomasochistic or dominant and submissive behavior as a regular part of their relationship. With one exception (the lovers in the triad), all had been in the relationship for at least one year. The duration of the relationships ranged from six months to 18 years, with an average of 5.5 years.

The respondents were all members of the Arizona Power Exchange (APEX), a Phoenixbased BDSM education, support, and fellowship organization. The first author identified committed couples and invited all identified committed couples to participate in the study. In one case, a respondent couple reported to their friends their positive interview experiences and recommended participation. One couple was obtained in this manner.

\section{Interview Process}

The first author presented the study as part of his doctoral dissertation. Each couple was told that interviews would be conducted with each member separately. Participants were then asked if they would be interested in being interviewed for the study. At the initial meeting, the research was explained, including the possible risks and benefits of participating and the potential for emotional damage or discomfort. If both members of the couple wished to participate, each partner was asked to sign an informed consent form. No couples declined at this point. The study was approved by Northern Illinois University's Institutional Review Board.

The interviews were conducted by the first and third authors. The first author took the lead in all interviews, and the third author took parallel notes and asked clarifying questions throughout when she felt it was needed. It was originally anticipated that some individuals would

\footnotetext{
${ }^{3}$ A collar is a symbol sometimes given to a submissive or a slave by the Dominant or Master in recognition of the power exchange relationship. Collars can be overt, such as a round steel neck band or a chain and lock, or subtle such as a necklace or ankle chain.
} 
be more comfortable answering questions directly to the third author, a submissive woman. It turned out that the third author's presence was sufficient in building trust and comfort in the respondents, and thus the first author was able to take the lead in all interviews.

All interviews were recorded on audio tape. The taping was presented as optional to the respondents, but none declined to be taped. The tapes were transcribed over the course of the study, and the transcribed answers were spot checked against the field notes.

The interviews with each individual were conducted using a variation of the techniques developed by Kinsey and his associates (Pomeroy et al., 1982). Using the Kinsey form of interviewing allowed the respondents to partly steer the interviews by providing information in a natural flow while ensuring that all the intended questions were covered. This format allowed for consistency in the questions asked and facilitated rapid recording of field notes to maximize focus on the answers and any nuances. After the first few interviews, the form was modified slightly to revise questions that required elaboration or that did not produce results. Subsequently, the first author went back to previous respondents and performed a follow-up interview to fill in potential gaps in the data.

The interviews started with simple questions such as age and occupation, then moved into lifestyle identification. Relationship issues were asked around the middle of the interview. The last question regarded rituals. This provided a forum for the respondent to discuss areas of the relationship that were particularly satisfying, thus ending the interviews on an upbeat note.

In some cases, a line of questioning was abandoned when it became clear that the questions were annoying the respondent. For example, this occurred during some interviews when asking a respondent whether he or she enjoyed taking on the bottom role for a series of BDSM activities. For respondents who exclusively top, saying "no" repeatedly grew tiresome. In those cases, the interviewer moved on to the next set of questions. Statistical analyses include the answers that were provided before the line of questioning was abandoned.

The participants' interview notes and recorded media were identified with only a couple number. These coded identifiers were the only link between the forms and the participants.

Most of the individuals in APEX were open to fellow members regarding their kink and most of these couples openly played at BDSM events. As witnessed by the first and third authors, many of the respondents in the current study had previously revealed very intimate details in group settings. During the interview process, the first author watched to see if he could detect any reluctance regarding the taping process. In two separate interviews he saw a single glance at the audio recorder. The glances were not overt, and thus it was decided to not acknowledge any potential issue and taping was continued. The apparent discomfort appeared momentary and the interviews continued on course. In both occasions, the glance occurred during the question regarding whether the respondent had any secrets from his or her partner. 


\section{Results}

The results are organized into seven parts: Demographics, Partner Selection, Relationship Description, Power Dynamics, BDSM Roles as Relationship Tools, Managing Boundaries, and Creativity and Adaptability. Illustrative quotes are labeled with role and gender but without age or other demographic information to protect the anonymity of participants. Given the close-knit nature of APEX, adding age or other demographic information to role and gender would have risked allowing participants to be identified.

\section{Demographics}

Table 1 displays the self-identification of participants as dominant, submissive, sadist, and masochist. As can be seen in the table, dominant and sadist were not synonymous. Likewise, submissive and masochist were not synonymous. Further, a substantial number of individuals identified in roles seemingly at odds with their relationship type. For example, although all 13 men in male dominant relationships identified as a Dominant, 3 also identified as a submissive, and 5 identified as a masochist. Sixteen out of 33 respondents had tried the complementary role at least once; six of these did not want to repeat the switching experience.

Table 1

Participants' Self-identified BDSM Roles

\begin{tabular}{llllll}
\hline Sex & Relationship type & Dominant & Submissive & Sadist & Masochist \\
\hline Men & & & & & \\
& Male Dom/female sub $(n=13)$ & 13 & 3 & 10 & 5 \\
& Male sub/female Dom $(n=2)$ & 0 & 2 & 0 & 2 \\
& Switch $(n=1)$ & 1 & 1 & 1 & 1 \\
\hline \multirow{2}{*}{ Women } & & & & & \\
& Female sub/male Dom $(n=13)$ & 5 & 13 & 4 & 10 \\
& Female Dom/male sub $(n=2)$ & 2 & 1 & 1 & 1 \\
& Switch $(n=2)$ & 2 & 2 & 2 & 2 \\
\hline
\end{tabular}

Note. The male participant who is in two relationships is included in the "male dominant" row as that is his role in his primary relationship. He is a switch in his other relationship. As with the other participants in the study in switch relationships, he identifies as a dominant, a submissive, a sadist, and a masochist.

Table 2 displays the demographics of participants, separated by sex. Participants reported a high level of happiness with their BDSM orientation (9.34 on a 1-10 scale).

All but one respondent thought a BDSM relationship was required or extremely important in their lives. That one stated that they could have found satisfaction in a more traditional form of $\mathrm{D} / \mathrm{s}$ relationship, such as being a nurse married to a doctor. When asked if they could leave the lifestyle, none said they could. 
Table 2

Participant Demographics

\begin{tabular}{lll}
\hline Demographic & Men $(n=16)$ & $\begin{array}{l}\text { Women }(n=17) \\
\text { Mean }(S D) \text { range }\end{array}$ \\
\hline Age & $47.8(8.8) 35-70$ & $40.4(8.8) 20-53$ \\
Frequency of sexual activity & $2.5(3.3) 0.1-14$ & $2.5(2.4) 0.1-7$ \\
Frequency of BDSM activity & $1.4(1.6) 0.25-7$ & $1.5(1.8) 0.04-7$ \\
Sex $\rightarrow$ BDSM & $52 \%(34 \%) 0 \%-100 \%$ & $55 \%(39 \%) 0 \%-100 \%$ \\
BDSM $\rightarrow$ sex & $60 \%(38 \%) 0.002 \%-100 \%$ & $68 \%(42 \%) 0 \%-100 \%$ \\
Frequency of masturbation & $2.2(2.9) 0-10$ & $4.5(7.6) 0-30$ \\
Frequency of solo BDSM & $0.3(0.9) 0-3.5$ & $0.5(0.9) 0-3.5$ \\
Number of sexual partners & $57.8(86.5) 5-350$ & $33.4(28.3) 7-100$ \\
Number of BDSM partners & $17.6(21.3) 2-80$ & $19.8(48.3) 2-200$ \\
Age of awareness of being kinky & $20.4(12.8) 10-52$ & $18.2(14.8) 5-53$ \\
Happiness with BDSM orientation & $9.0(1.0) 7.5-10$ & $9.6(0.6) 8-10$
\end{tabular}

Note. Frequencies were times per week. Sex $\rightarrow$ BDSM refers to the percent of the time that the participant does BDSM when they have sex. BDSM $\rightarrow$ sex refers to the percent of the time that the participant has sex when they do BDSM. Masturbation data include one man who reported that he masturbates 10 times per week, one woman who reported twice daily, and one woman who reported masturbating 4 to 5 times per day. One female participant answered that she "always knew" she was kinky but did not provide an age. Happiness with BDSM orientation was measured on a 1 to 10 scale with higher numbers indicating greater happiness.

\section{Partner Selection}

A series of interview questions focused on the origin stories of how the couple met, what caused them to explore more deeply, and what motived them to commit to this relationship.

\section{Meeting the Partner}

When it came to the stories of how they met, there was $100 \%$ congruity between the partners' reports as to the circumstances of their meeting and very high congruity as to the details. Frequently the individuals within the couple would use the same phraseology. It is apparent that the couples have told and retold the stories of how they met and have bonded to the point where their mythology is an important part of their relationship. Eight out of the 17 couples first became aware of each other through the Internet, a very high proportion for the 1990s (Rosenfeld, Thumas, \& Hausen, 2019).

Submissive Woman: I answered his Yahoo personal ad. "Loving dominant seeks little miss submissive." And the first thing it said is, "A submissive is not a doormat. She is a gift. The submission is a gift. I will respect her limits." I found BDSM personals on the net - must have gone through three hundred in one night - just screaming through them and most of them were "Come suck my cock; you will be my slave; yada yada yada." Either that or they were really wimpy. And then I find this one. It is very honest and open. Four days later we met in person.

\section{Partner Attraction}

When asked what was the initial attraction to their partner, the answers included: lifestyle interests or demeanor (15 out of 33), physical (9), "energy" or presence (7), honesty (6), playful (1), taboo (1), and humor (1). The transcripts of the interviews show that these values were 
important even if they were not named at the time of the specific question. When asked what was it that made the partner "the one," the answers included: lifestyle intent (10), "chemistry" (7), honesty or trust (5), friendship (2), playful (1), and physical (1).

A number of the respondents reported stories of having been in a vanilla relationship before, having been unhappy, and being committed to having a BDSM relationship where their needs could be met.

Dominant Woman: How I really got into it was when we first started dating, he said, "Look before we go any further, I have these things about me you really need to know." And he sat and told me, and he was so terrified, and I sat and started laughing, not at him, but oh thank you god for sending this wonderful man to me.

Dominant Man: But we've always had good communications even from the very first, from the very first day. I didn't have a play bag with me - a soft cowhide belt I had on. And I whipped her with it. And after I finished, she laid back on the bed and she had this huge sigh and she had this wonderful look - which I've grown to love - and she says "Thank God, I was afraid you weren't going to do it hard enough." That was what I think one of the most endearing lines she ever said.

Just as couples had a well-established "how we met" stories, a number of couples have a well-established "why we were attracted to each other" stories.

Submissive Man: I thought she was very pretty. She let me touch her. While we stood in the garage and revved up the Harleys. At my buddy's house on New Year's Eve, here's this girl I didn't know, and she cuddled right up to me and I got me a handful of heinie and thought, "What could be better than this?"

Dominant Woman: What attracted him to me was I had this one-piece jumpsuit on and I had a pair of shoes on that had really high heels and I was looking like a fox. We were out there in the garage listening to the bike roar and vibrate, and he reached over and touched my ass. And he said "Ohhh" and I let him touch it, "cause I knew I wanted him. And that was it.

\section{Relationship Description}

This section explores the roles identified by the respondents and their partner, the level of compatibility between the partners with their D/s and BDSM needs, what they see as working in their relationships, what issues they see, and how they handle differences.

\section{Roles}

When asked to briefly define their role and the role of their partner, answers within each couple were largely congruent with a few exceptions. Two submissives referred to their partner's role as a Master. No Dominant used that term. In one couple, the Dominant referred to their partner as a "slave," the submissive used that term for themselves and used the term "Master" for 
their Dominant. Later in the interviews the respondents were given the opportunity to define their roles in detail. These responses were quite specific, varying from couple to couple.

Submissive Man: I tend to define myself in a first comment as being an ass kisser and a boot licker. I am a submissive to women, I am subservient. I'm not a masochist. I don't like intense pain. I enjoy cross-dressing a lot. I enjoy wearing clothes and it puts me in a different persona that is always submissive.

Switch Woman: I would say we only have well-defined sexual episodes of power exchange. Because for me it's VERY important that it's egalitarian. I think that's because he is a truly egalitarian male. I think that's one of the reasons we got together.

\section{Compatibility with Dominant/submissive and Sadist/masochist Identifications}

The respondents were asked to name their primary role, and then asked if they were Sadistic, Masochistic, Dominant, and submissive. During the interviews, the responses were coded by the first and third authors as identified with that role, indifferent to that role, or explicitly not identified with that role. Thus, each participant was coded as identified with, indifferent to, or not identified with each of the four roles (Sadistic, Masochistic, Dominant, and submissive). Many participants identified with multiple roles including seemingly opposite roles (e.g., a participant identifying as both Dominant and submissive, or both Sadistic and Masochistic). The four switches, in fact, identified with all four roles.

A couple was considered incompatible on a particular axis if one partner was positive on the role and the other partner was negative on the corresponding role. For example, a couple would be considered incompatible on the $\mathrm{D} / \mathrm{s}$ axis if the male partner identified as dominant, but the female partner rejected the identification of submissive. Thus, compatibility could consist of both partners endorsing corresponding roles (e.g., a dominant-identified woman involved with a submissive-identified man), both partners rejecting corresponding roles (e.g., a man who rejects a sadist identity involved with a woman who rejects a masochist identity), or one or both partners being indifferent to the corresponding roles (e.g., a sadist-identified woman involved with a man who is indifferent to the masochist role).

The 15 non-switch couples showed 100\% compatibility along the D/s axis aligned with their relationship orientation. That is, all 13 men in the male dominant/female submissive couples identified as dominant, and all 13 women in the male dominant/female submissive couples identified as submissive. Likewise, both women in the female dominant/male submissive couples identified as dominant, and both men in the female dominant/male submissive couples identified as submissive. These 15 non-switch couples showed only $67 \%$ compatibility along the $\mathrm{S} / \mathrm{M}$ axis aligned with their relationship orientation. That is, for 10 couples the dominant's identification (or lack of identification) as a sadist was compatible with the submissive's identification (or lack of identification) as a masochist, but 5 couples were incompatible $(2 / 5$ showed a sadistic dominant in a relationship with a specifically non-masochistic submissive, $3 / 5$ showed a masochistic submissive in a relationship with a specifically non-sadistic dominant). 
Thus, successful long-term dominant/submissive relationships did not appear to require sadists to be matched with masochists (or masochists to be matched with sadists).

As noted above, the four switches identified with all four rules. Thus, the switch couples showed perfect compatibility on both the D/s and S/M axes.

\section{Compatibility, Perceived Compatibility, and Knowledge of BDSM Interests}

Respondents were asked if they were interested in 32 typical BDSM activities from the top perspective and from the bottom perspective. During the interviews, responses were coded by the first author as $2=\mathrm{I}$ really like it, $1=\mathrm{I}$ like it, $0=$ neutral, $-1=\mathrm{I}$ dislike it, $-2=\mathrm{I}$ really dislike it. Participants also reported their perception of their partner's interest in the activities.

Compatibility of BDSM activities was calculated as the correlation between one partner's 64 responses and other partner's 64 responses with responses paired so that the top perspective for one partner was paired with the bottom perspective for the other partner. Across couples, compatibility ranged from .05 to .78 , indicating that all couples showed a tendency to align (albeit some weakly) on which activities they liked and which activities they disliked. Average compatibility $(M=.36, S D=.19)$ was significantly above $0, t(16)=7.75, p<.001$. It should be noted, however, that the significant level of average compatibility does not mean that couples had no areas of disagreement. Nearly all couples (16/17) had at least one activity that one partner liked but the other partner disliked. Compatibility did not differ across relationship type (male dominant, female dominant, switch), $F(2,14)=0.04, p=.96$. Relationship duration was not a significant predictor of compatibility, $b=-0.000, t(15)=-0.03, p=.98$.

The likelihood that a modest level of compatibility would be workable is contrary to the conjecture by John Money (1986) that so-called "paraphilic" relationships were unlikely to be successful as there would be little probability that there would be a perfect match between complementary interests. The present data illustrate that successful long-term couples do not require a perfect match between interests.

Perceived compatibility was calculated as the correlation between one partner's 64 responses and that partner's perceptions of the other partner's interest in the activities. Perceived compatibility ranged from .07 to .69 . Average perceived compatibility $(M=.39, S D=.17)$ was significantly above $0, t(16)=9.49, p<.001$. Perceived compatibility did not differ across relationship type, $F(2,14)=0.09, p=.91$. Relationship duration was not a significant predictor of perceived compatibility, $b=-0.002, t(15)=-0.30, p=.77$.

Knowledge of partner's interests was calculated as the correlation between one partner's perceptions of the other partner's interest in the 64 activities and that partner's actual interest in the activities. Knowledge of partner's interests ranged from .35 to .80 . Average knowledge $(M=$ $.63, S D=.10$ ) was significantly above $0, t(16)=24.91, p<.001$. Knowledge did not differ across relationship type, $F(2,14)=0.31, p=.74$. Relationship duration was not a significant predictor of knowledge, $b=0.002, t(15)=0.51, p=.62$. 
Summarizing the above, the couples had a significant but modest level of compatibility of interests (average $r=.36$ ), an accurate perception of their compatibility of interests (average $r=$ .39 ) and a high level of knowledge of their partner's interests (average $r=.63$ ). The accuracy of the perception of the compatibility of interests is further illustrated by a significant correlation between compatibility and perception of compatibility, $r(15)=.71, p=.001$.

It was expected that knowledge about their partner's BDSM interest would increase over time. However, relationship duration was not a significant predictor of knowledge. This suggests that most of the disclosure by the partners happens early in the relationship. It was also expected that compatibility would increase over time based on the idea that couples would, over time, adopt some of the interests of their partners, but the data showed no effect of relationship duration on compatibility.

\section{What Works}

When asked what works in the relationship, respondents listed: communication (19), mutual support (14), openness or honesty (9), lifestyle (7), physical/sexual attraction (4), humor (3), love (2), religion (1), and intimacy (1).

Several themes appeared frequently in the interviews. The respondents held the perception that dominance and submission directly relate to intimacy. There is a high value placed on communication and full disclosure within the couple. There is pride in being able to read their partner and to explore needs until both parties have their needs met. Throughout the interviews, the respondents expressed pride in their relationships and pride in their partner. In the respondents' descriptions of themselves and their partners, there were no indications that one role might be considered better than another.

Submissive Woman: There's not real boundaries as far as walls that are constructed of things that we can't do or shouldn't do. There's this tremendous sense of openness. And that deals with our sexuality; that deals with just about anything. If it's on my mind I say it.

Submissive Woman: I'm teaching my partner how to bite. He comes from a very nice family, very good Norman Rockwell school of BDSM type thing. And I'm like "Bite me" and he's like "What? But it'll hurt." Yeah.

The very close connection of some of the respondents extended well beyond BDSM:

Submissive Man: When we met, both our now former marriages were on the rocks. So together we crawled into a bottle of booze and drugs and partied and carried on for ten years. And after one particular binge, she said "I gotta go to one of them meetings." I stopped drinking right away because I didn't want to encourage her. So, I quit drinking in support of her. We got drunk together, so we got sober together. 
Issues

Fourteen participants reported BDSM-related relationship issues: defining their BDSM relationship (6), wanting the partner to be more aggressive or "Toppy" (6), more play time (2). Other issues included better communication (8), polyamory (6), wanting to change partner's habits (5), aging and health (3).

Submissive Woman: My biggest concern is he may never be able to be the dominant he hopes to be. My every hope and dream is that he is. But I need someone strong in my life. That is a concession I will have to make if I need to. Cause I'm not changing partners.

Submissive Woman: There's a little bit of insecurity thinking, can I fulfill all his fantasies or enough of them to keep him interested for a lifetime, or is there going to be the new better submissive model that'll come through?

Dominant Man: To be a good top or dom takes not only some physical energy, it takes a great deal of mental and psychological energy. And sometimes all that stuff, it's just hard for both of us to get there. And that's probably more my responsibility than hers because she can flip into sub space without the same energy expenditure that it takes me to be purely in top space.

When the respondents were asked what they wanted modify in themselves and in their partner, they listed issues such as losing weight ( 24 for self, 16 for other), being better as a partner such as being in tune with their partner's emotions (19 for self, 15 for other), being better at their BDSM role (26 for self, 12 for other). For example, one participant explained:

Submissive Woman: I wish my beloved partner was a bit more sadistic to get the adrenalin going, but it's still so new so I ain't pushing. Right now, he has a real hard time inflicting any pain on me, even if I'm going "Oh god please, help me, give me some more -now".

\section{Handling Differences}

In handling differences, communication was the most common factor mentioned. With the exception of two respondents (both from the same couple), all respondents discussed communication in either their answer to the direct question regarding handling differences or elsewhere in the interview. In response to this question, respondents listed: communication (23), using counselors (13), arguing or getting noisy (8), using the power dynamic to handle (6), not rushing the process / setting-aside until later (6), letting it sit / agreeing to disagree (2), succumbing / conceding (2), and walking away for the moment (2).

The following quotes demonstrate rather sophisticated self-management and communication tools being used by these couples.

Submissive Woman: If Sir says something and my gut reaction is pain and my other gut reaction is "He did that on purpose." I have an immediate counter-reaction that says, "He 
would never do that on purpose to me. He's claimed me - not to hurt me." It's always made me step back from any intentionality of the pain, so that I could say, "I think I misunderstood because this is what I heard you say and this is what I thought you were saying, you know, hurting." And he recognizes, "no." And so it allows us to get down to a communicative level.

Dominant Woman: We've like "Okay I want to go out for coffee, and I want to talk to my best friend." And so, my partner's cross-dressing persona comes out, we sit there and we talk, so that we're best friends first and we love each other very deeply so that everything else works well.

Submissive Woman: He was always a little bit more reserved than me, so it took a little while to get him used to talking. He was afraid of even opening his mouth because in previous relationships the girl would bolt. I said, "I'm not going anywhere. Tell me anything. You know, yell at me. Try not to, but I will take it in stride, and we will talk about it."

Of the 13 respondents who had been to counselors at some time in their life, two used the counselors to get more comfortable with their BDSM interests. None had gone to counseling to be "cured" of their interest. Four respondents had sought out a kink-friendly counselor.

\section{Power Dynamics}

The respondents were asked a set of questions designed to reveal the scope and boundaries of their power exchange. Areas covered included the domains of power transfer, power in sex and BDSM, role reversal, punishment, and a sense of ownership or being owned.

\section{Power Domains}

Participants were asked a series of questions regarding power in daily life (Who is the primary earner? Who does the dishes? Housework? Laundry? Who makes the major choices? Initiates sex? Initiates SM? What domains do you have personal power?). Regarding finances, in eight couples the man is the primary breadwinner, in two the woman is the primary breadwinner, and in seven expenses are shared relatively equally. There is no pattern as to income source and power dynamics between Dominant and submissive roles.

Twelve of 17 couples report that the Dominant has control over the submissive in some form in daily life, two couples report that any control is only for the duration of any play scenes, and three couples disagreed as to whether the power exchange extends into daily living. In all cases of disagreement, the submissive said the Dominant has control in daily matters.

Submissive Woman: When it came to the house, we went through a whole process together talking about how we'd do it. We both put a lot of input in and then Sir said "Okay, this is how we're gonna go then." He listens so closely to what my needs and my wants are. He makes sure that whatever is going on is meeting both of our needs and 
some of our wants.

Submissive Woman: I told him from the first letter that kids were non-negotiable - I'm not having them. And he was in the gray area about that; but once he started thinking about it, he told me that he felt he was too old and also not set up for children. So that's when we had my tubes tied.

In describing their areas of personal power, Dominants' responses included: sex (9), total control or final say (7), dress (4), punishment (3), finances (3). Submissives responded that their Dominants had power in all areas (6), finances (4), dress (4), outside activities (3), and sex (2). When asked to describe their personal areas of power, submissives' responses included: outside work (6), personal or family finances (6), homemaking (4), relationships with kids or family (4), major purchases (2), ability to voice opinion (2), food (2), healthcare (1), dressing the Dominant (1). Dominants responded that their submissives had control of: healthcare (6), job (6), personal or family finances (5), relationships with children or family (4), veto of BDSM activities (3), dress (2), household (2), day-to-day life (2).

Overall, Dominants and submissives were largely in agreement: there was substantial congruity as to who had the power with no major areas of contention for any of the couples. Answers also revealed that submissives had considerable agency in their lives.

There was good agreement (88\%) between partners as to whether the couple was in a 24/7 (full time) Power Exchange relationship. It should be noted that many of the couples went on to define 24/7, saying that of course there are dishes to wash and children to feed, but 24/7 means there is at least a background BDSM presence in their lives on an ongoing basis.

\section{Power Fluidity}

In all couples, a common thread is the appearance of a power exchange in BDSM scene space. That is, power exchange occurred during BDSM activities for all couples, even if power exchange did not extend into other areas of life. Frequently respondents talked of absolute control in the scene. At the same time, there were detailed discussions about the Top taking care of the bottom. Thus, even though the Top exerted control over the bottom during BDSM activities, the Top also took care of the bottom - control and caretaking were not mutually exclusive. In most couples, the dominant / submissive nature of the relationship made the boundaries between BDSM power exchange and the power dynamics of daily living very fluid.

Submissive Woman: I have a fair amount of personal power in the lifestyle in the sense that I am allowed to voice my opinion and it is taken under consideration. He has the final say, but if there's somebody I would like to play with or not like to play with, or if I have feelings that I don't want him to play with somebody, I can state my case. He'll listen. I've said there's absolutely no way I want to play with so-and-so. He respects that.

Dominant Man: In terms of power issues lifestyle related, I pretty much retain all the power there. I choose to allow my partner to be a little less constrained than some doms keep their submissives. That being said, I exercise absolute control over who she plays 
with, or if she plays with someone. If it's someone that I'm uncomfortable with and she might want to play with somebody, my "No" is an absolute. She would never think of doing that without my permission.

\section{Switching Roles}

It might be confusing for an uninformed bystander to watch the Dominant tell the submissive, "flog me." This study found that such combinations are not uncommon. Five out of 13 dominant men and 1 out of 2 dominant women considered themselves masochistic, while 3 out of 13 dominant men and 1 out of 2 dominant women considered themselves submissive. In some cases, the nature of the relationship (e.g., 24/7 Dominant/submissive) meant that this "opposite" role might not manifest in that relationship. In other cases (most notably in the switch relationships), all roles might manifest at one time or another.

Dominant Man: I've participated in certain submissive activities but generally not in a submissive role. I tend always to be in the controlling role of it. One of my favorite activities are water sports and sometimes I find it satisfying to be on the receiving end.

Dominant Woman: I am a dominant that bottoms. More masochistic, some submissive tendencies. I think that I find it really hard to be submissive because I might become submissive. I had a relationship - it wasn't in the S\&M community, but the last relationship I had was a real power struggle. That man had me where I couldn't even plant flowers without asking him where. It wasn't consensual, is the thing. I didn't realize it was happening, till it was already happened. That's why I find it really hard to be real submissive. But I do bottom.

\section{Atonement}

Two couples discussed punishment as a method of addressing transgression. For these couples, punishment provided atonement and enabled the couple to fully move past the offense.

Submissive Woman: I'd say I've been punished twice in three years. It involves a single swat. But it's a ritual in and of itself. We discuss the offence totally before the actual punishment. And then it's as much of a mental place as it is one physical swat. Punishment for us can only result if: one, we've already discussed something; two, we've covered it and I already know what my behavior is supposed to do; and three, I specifically didn't act on that. It's only happened twice. With the punishment also comes the absolute total ending of that event never to be brought up again.

Dominant Man: Punishment is a cleansing act. It's only done when there's been a serious offense and the punishment marks the end of that offense. She's released of her responsibility from and it doesn't exist in our life anymore as of the culmination of the punishment. I only punished her once. 


\section{Rituals}

For the final question in the interview, the respondents were asked to describe the rituals they had in their relationship, both one-time rituals marking special occasions and ongoing rituals. The placement of the question was to allow the individual to remember good things about their relationship, thus leaving the interview with a validating experience of the event. Responses also provided insight into "bonding moments" within the relationship.

Rituals were used to return the partners to their BDSM roles, to reinforce those roles over time, and to maintain the bond between partners. These rituals were initiated as often by the submissive as the Dominant. There was often a quality in these rituals that expressed the excitement of preparing to be with their partner.

Dominant Woman: One ritual is when he brings me something, he just sits and holds it until it's time for me to get it. And sometimes I'll see it there for ages and I just sit and allow him to hold it because I know that that's a real special thing because it's his nature to jerk on me and say "here, here, here," "I've got it, I've got it" and it's my nature to say "I want to take it right this second." But we both just sit there, and he holds it and prolonged the moment because it's not typical of us, and it's so sweet.

Dominant Man: When I am done taking a shower she is there, holding my towel for me. She presents the towel, saying, "Your towel, Master." It warms the cockles of my heart it reminds me how much I love her.

The respondents also described one-time bonding rituals. These were frequently a collaring ceremony, in public or in private.

Dominant Man: The collaring actually preceded the marriage by a number of years. That was a one-of-a-kind ritual that occurred with her tied to a tree in the middle of the forest. So, it was a very special and something that we never attempted to re-create because it was just too perfect of a moment.

\section{BDSM Roles as Relationship Tools}

The respondents discussed their chosen BDSM roles as tools for supporting their relationship in three areas: getting into their relationship headspace, in initiating sex and BDSM play, and in dealing with conflict.

\section{Getting into Headspace}

A number of the respondents highlighted the distinction between their roles in the larger world (employee, parent, relative in their birth family, etc.) where they had to alter themselves to be effective and/or safe in the outside environment and their roles in their relationship. When returning to the relationship, they took time and effort to make a transition to their declared role. Respondents often referred to their private world as the "real world" - it was apparent that they saw their relationship role as their real identity. 
Dominant Woman: The clothing helps me with my persona. It's hard for me to be in the dominant mode when I'm wearing bobby sox and gym shoes and jeans. I just can't get into it. I have to have something on sensual that triggers me into my other persona.

Dominant Man: D/s has its own language and that is really important when we reconnect. She wears clothes at work. When we reconnect, she's not allowed to wear clothes. And so that is a visible signal to both her and I that we know we're not at work anymore and now it's time for you to take care of me. The removal of her clothing is a ritual that says, "This is what part you're in."

\section{Initiating Sex and BDSM Play}

To get sex or BDSM play started, either partner might initiate a ritual that clues the other. Respondents reported that they feel like they are flirting when using such rituals. Kneeling is one of the symbolic acts frequently used in various BDSM rituals, including initiating BDSM play.

Dominant Woman: We have other rituals, like when we play, we always start it with I walk in and he's on his knees, on the floor, waiting for his collar.

Submissive Woman: All I have to do is kneel, and he has a hard time walking. <laughs> I'm serious. This man has the best circulation system. His spongiosum works constantly. All I have to do is hand him a slapper or hand him the flogger or - first time I called him Master, scared the hell out of me. He came running into the bathroom. I swear to god he was like happy and halfway crying and got a hard-on and the whole nine yards. This is what he tells me, and I see it in his eyes -he is so honored that someone like me who wants to kneel and submit to this man. I've tried almost damn near anything I've ever wanted sexually, so the fact that I would choose him just gives him a raging hard-on. And I just love it. I am so flattered.

Submissive Woman: He likes face slapping. And with my history of abuse that was a real, real hurdle for us. But we can go there fairly comfortably now. I can think of a couple of times I initiated it because it just felt really right in the way everything was going. I put his hand up to my face. Open palm up to my face. And he knew what I meant.

\section{Dealing with Conflict}

The language and rituals of the BDSM relationship frequently were used when there was conflict. The rituals and roles seemed to simplify the conflicts and kept both partners out of egobased stands. The couples also reported that they sometimes use naturally occurring conflict to clear up long-standing issues or to reconnect with each other.

Dominant Man: One time in particular that REALLY stands out in my mind. She was getting in my face about something, and I was being my typical self - I tend to kind of turn everything in. She was getting in my face and getting very - and she - I don't want 
to say stepped over the line - but she $<$ ahem $>$ pushed the right buttons and I snapped. I don't think I said kneel, I think I was just like $<$ he sharply points down $>$ on your knees, now, here. And it was like < finger snap $>$ boom, she was on her knees right there with her head down. And it was like "Whoa!" < laughs> It's like "Oh boy, that felt good."

Submissive Woman: He has found that I can be in like full rowdy-ass mode and all he has to do is go "Kneel." And I'm right there. And he got a hard-on off of it. And he's like, "Omygod it works." And it's like, <very quietly> "I told you." He forgets - as many people who deal with me forget - that all you have to do is say one word, and I'm there.

\section{Managing Boundaries}

Many of the couples engage in BDSM with other individuals. Some also engage in external romantic or sexual relationships. These erotic interactions outside the dyad highlight the benefits and challenges of non-monogamy and the ways that couples manage jealousy.

\section{Non-monogamy}

Some of the respondents spoke of issues regarding their explorations into polyamory, a relationship form in which individuals have more than one romantic partner which usually includes sex and, for the BDSM community, BDSM. Some respondents spoke of being willing to work with polyamory as a gift to their partner.

Submissive Woman: We have learned over the years to be very selective about who we invite in close. And we are very discerning about submissives who would try to insinuate themselves between the two of us emotionally, or a dominant who would try to do the same thing. We enjoy playing with other people, but they have to be the right people and they have to be people that respect our relationship.

Submissive Woman: I think there's a certain level of gift giving when we're incorporating other partners into our play. I would say me being okay with the fact that he's gonna play with other people - although he definitely has the right. I see that as being a gift to the submissive and somewhat a gift to him. But I have the same opportunity on my end if I have permission. I think the level of trust that we have is a gift that we give to each other. Because we walk a much finer line than in a conventional relationship.

\section{Jealousy}

Respondents discussed two topics related to jealousy: the boundaries they placed on their relationship (e.g., what behaviors are expected to be exclusive to the relationship) and how the couple handles jealousy when boundaries, expressed or otherwise, get crossed.

Switch Man: My partner and I do have boundaries. The vast majority of them are sexual although they don't necessarily mean no genital contact. I can do scenes where I've done 
clothespins on men. I had this scene where a friend was considering having a group vaginal fisting done on her, and I had interest in participating in that. My partner and I talked about it. She's like, "Well, it's like, god, you have your hand inside her vagina. I mean that seems pretty sexual. But on the other hand, it's fisting," and so that's where we sort of negotiated that that would be okay. Isn't it interesting? Isn't sex interesting? You know it wouldn't have been okay for me to finger fuck her, but it would be okay to actually fist her.

Dominant Man: I used to do a fair amount of piercing of other people. It was very important to me when piercing a woman that her partner be there, and I let her partner be at her head. So, you guys - I'm doing the piercing - but you guys are doing the bonding. The possibility that the woman would bond with me after this intense experience felt threatening to me. It was actually for the self-protection.

Submissive Woman: We went to a friend's dungeon party. One of the women there had what I thought were physical attributes Sir was always attracted. And I felt Sir was paying attention in a very particular way to that woman which is the first time in four years that his attention has ever been on anyone except me. And that was okay, except he stopped touching me in the ways he usually does when we're out publicly with people. So, he was focusing on what he could do with those boobs. It was an unusual experience for me. That's where "Let's rethink about multiple partners." It was fine in theory but to have my first experience of it was like "Whoa let me rethink this."

\section{Creativity and Adaptability}

Throughout the interviews, a constant theme emerged of people finding creative ways of meeting their own and their partner's needs. The commitment to mutual transparency, communications, and the practice of paying attention to each other's needs led to many examples of respondents adapting to fulfill each other's needs and desires.

Switch Woman: I had not a whole lot of interest in receiving pain initially. Like with the nipple thing -reading the Beauty books which in some parts they did some things with clamps and bells and I was like "Oh, that's kind of interesting, but-" So there was like a little bit of interest but it wasn't like a thing. And now we do that like a lot. I think he's trained me to respond to it or something. <laughs $>$ It's that whole Pavlov's dog thing. It really does work, well. Well, and like having something done that you don't like while having an orgasm can - eventually you end up associating the two.

Submissive Woman: One time I grabbed the rabbit flogger and just went at him. And he's just like < her eyes widen>. So, I went and got him one and I said, "Come on; let's go." So, we basically had like, I stick my butt out - he nails me and then I turn around and, whack, and nail him back. And of course, I couldn't hardly hurt him at all with that rabbit flogger. After the first ten minutes, his face was like "I don't believe you're hitting me. But after he got through realizing that I was not topping him, he FINALLY HIT ME. And it was just like < sharp inhale through nose $>$ okay, that one sunk in, do it again. It was fun, because he'd never really let himself go that much. It's like a pillow fight with 
floggers.

Dominant Man: [W] hat pushes my button is what pushes my submissive's button. I can find enjoyment out of a wide variety of activities with a person who's into them and finds them exciting. So, if a person gets really excited and turned on by being spanked, spanking that individual would be a real button-pusher for me.

\section{Discussion}

Voices from within and outside the BDSM/Leather community have argued that longterm power exchange relationships are very likely to fail. From within the community, Townsend (1972) cited the incompatibility between satisfying SM and romantic affection. From outside the community, Money (1986) cited the difficulty of finding a perfect match of fetishes and interests. The present study emerged from the first author's observations of what appeared to be successful long-term relationships within the membership of the Arizona Power Exchange, the fifth oldest pansexual BDSM club in the United States. Thirty-three individuals in 17 longterm BDSM relationships participated in long-form interviews conducted by the first and third authors. The interviews used a Kinsey-style format that provided freedom for the conversation to flow naturally while ensuring that all questions were covered.

\section{Long-term BDSM Relationships}

At its most basic level, this study demonstrates that quality long-term functioning relationships do exist for BDSM-oriented couples. The sample included successful male Dominant/female submissive relationships, successful female Dominant/male submissive relationships, and successful male/female switch relationships. Some of the relationships (12 out of 17) have a high degree of apparent control of the submissive by the Dominant in daily living. Others restrict their power exchange to BDSM scenes. Across the couples with a high degree of power exchange, there was considerable discussion of the Dominants having power, but no discussion of the Dominants being better, or smarter, or of more value, than the submissives. We conclude that the surrender of authority by the submissive does not diminish the individual.

The respondents' descriptions demonstrate the multifaceted nature of BDSM. Many of the respondents had an active BDSM history over many years. They became well educated as to the possibilities and had discovered the roles and activities that suited them. Most considered the exploration of BDSM to be an ongoing process and expected that their interests would continue to grow and change. The respondents placed great value on their BDSM interests and activities, reporting benefits of the BDSM lifestyle that included personal growth and self-awareness, contribution to their relationship, a connection to the BDSM community, and better sex.

Many respondents told of having not disclosed their BDSM interests when they were in previous relationships, and of the dissatisfaction that came with the circumstances of those former relationships. All respondents stated that having a BDSM, or some other form of D/s relationship, was very important in their lives. These successful relationships came from early and thorough disclosure of the respondents' BDSM interests to their then prospective partner. 
In the process of partner selection, all of the respondents were aware that their prospective partner had BDSM interests prior to pursuing that partner for a long-term relationship. There was high congruity between the partners on how they met and how they chose each other. Partners often independently used the same phraseology in describing their meetings. These "getting to know you" stories were told with pride and had apparently been told many times before. These stories of "how we met" and "how we fell in love" indicate that a common mythology was important in the couple's identity. Half the respondents said that the lifestyle interests of their prospective partner were the biggest initial attraction.

\section{Relationship Values}

\section{Communication, Transparency, and Trust}

The most consistent relationship value voiced by respondents was communication, with statements that successful BDSM required open communication and disclosure. This includes the concept of transparency - described as full disclosure of emotions and needs. Respondents frequently told, when they perceived their partner had an issue, of being unwilling to let go until the issue was communicated and worked through. They considered resolution of issues to be more important than any possible embarrassment or discomfort to themselves or their partner. Respondents valued their ability to read their partners; they had invested considerable time and effort paying attention to their partners and learning what they liked and disliked.

The couples disclosed the details of their BDSM interests very early on in their relationships, and changes in interests were disclosed as they were discovered. Couples had a commitment to the personal growth of both partners as individuals. The mutual caregiving in these relationships stands in stark contrast to the troubling examples discussed by Pitagora (2015) of abusive relationships masquerading as consensual BDSM relationships.

In the power exchange model used by some of the BDSM community, the major negotiation is made up front when the individuals consent to and commit to their roles. Subsequently, the Dominant makes the final decisions. These decisions are not always in the submissive's favor - the Dominant may make choices in favor of his or her own self-interest. In order for the dominant/submissive model to work in the long term, however, the needs of both partners must be included. Further, even in the most stringent hierarchical relationships in the study, the submissive had the opportunity to state their wishes. We do not have data, however, regarding how often the Dominant then made a choice in line with the submissive's wishes.

\section{Rituals and Protocols}

The use of rituals in good times cemented bonding and had a flirtation-like quality. The use of rituals and protocol in difficult times returned the partners to a state of connection to each other, and this re-established bond helped the partners resolve the present upset. Thus, rituals provide these individuals a safe, structured way to interact so that conflict doesn't damage their relationships. It is notable that the use of deeper protocol when conflicts arise is contrary to the prevailing community rhetoric that advises dropping out of role to solve relationship conflicts. 


\section{Submissives/Bottoms Want More}

The respondents reported an ongoing theme of the submissive wanting to push the relationship harder and deeper in both BDSM play and in the power exchange dynamic, with some hesitation by the Dominant, who expressed care, caution, and an awareness of responsibility for their actions. This manifested as a consistent pattern in the interviews. Whenever there was disagreement over how much BDSM play or control should exist, the bottom or submissive generally wished to play harder or to define the relationship as more restrictive than the top or Dominant. Sagarin et al. (2009) reported a similar pattern in a study of BDSM scenes in which "bottoms expressed significantly greater anticipation of the evening's scene compared to Tops" (p. 190). These data suggest that society's image of the bottom being a "victim" is inaccurate; bottoms are at least as willing a partner in BDSM activities as Tops.

\section{Match of Interests}

The Dominant/submissive couples in the study showed perfect compatibility in terms of dominance and submission and positive but weaker compatibility in terms of sadism and masochism. Likewise, the couples showed significant but modest compatibility in terms of specific BDSM interests. It appears that compatibility regarding power exchange was more important for couples than compatibility regarding S\&M or regarding BDSM activities. Finding a complement in BDSM interests was part of the partner selection process but finding "the perfect match" was not a necessity for a successful relationship.

All respondents state that their BDSM orientation is built into who they are - their orientation cannot be changed. They did, however, demonstrate considerable fluidity within the BDSM arena, being able to acquire new tastes and being able to shelve some old ones when those interests did not fit with their partner. One process that appeared to facilitate this flexibility was the tendency for respondents to get turned on by their partner's excitement. So, although respondents did not necessarily directly internalize their partner's fetishes, they indirectly enjoyed those fetishes through the excitement and pleasure the fetishes provided to their partner.

Six out of 15 dominant men and women also identified as masochistic, and it was not uncommon for the Dominant to order the submissive to apply stimulation to the Dominant. The power exchange was defined by the respondents according to who did the choosing, rather than who received the stimulation. Thus, the Dominant was able to have their masochistic desires satisfied by being flogged by the submissive, while not losing authority within the relationship.

\section{Implications for the Understanding of BDSM Relationships}

As noted above, Hammack et al. (2019) provide a four-part framework for understanding BDSM relationships. The present data both support and challenge Hammack et al.'s framework.

The first element of Hammack et al.'s (2019) framework states that BDSM “relationships are typically characterized by recreational power exchange" (p. 576). The 17 relationships in the present study were all characterized by power exchange ranging from egalitarian couples who exchanged power solely in the bedroom or the dungeon to $24 / 7$ couples who exchanged power 
on an ongoing and pervasive basis. However, most of the couples would likely reject the term "recreational" as a descriptor of their power exchange. The power exchange, particularly for the dominant/submissive couples, represented a fundamental aspect of their relationship, providing role clarity, security, and tools for communication and the resolution of relationship conflict. To be sure, these couples also derived recreational benefits from their power exchange such as joy, fun, and stress relief. But the totality of the power exchange extended well beyond recreation.

The second element of Hammack et al.'s (2019) framework states that BDSM relationships "are consensual and collaboratively scripted" (p. 576). As with the BDSM community as a whole (Pitagora, 2013), consent lay at the core of the relationships in the present study. Further, the relationships could be accurately characterized as collaboratively scripted. Respondents were well aware that they had co-constructed their reality - that concepts such as ownership and slavery do not fit the "normal" world, and work for the relationship only so long as both parties are in agreement. As part of the co-construction, the respondents frequently used rituals to establish their roles, to bond with their partner, to initiate BDSM play, to work through conflicts, and to take care of each other. The co-constructed reality model of Berger and Luckmann (1966) appears to hold true for the studied BDSM relationships. Relationship partners, through the use of language, describe the nature of their relationship and repeat that description to each other so that, over time, the description becomes the reality for the couple.

Hammack et al.'s (2019) third element, the embeddedness of a BDSM relationship "in a larger community of shared meaning and practices" (p. 576) is one area in which the couples in the present study might differ from couples today. At the time of data collection, the couples were all members of the Arizona Power Exchange, a "community of shared meaning and practices." But many of the couples met and formed their relationships in relative isolation. BDSM couples today seem more likely to be connected, in person or via social media, to a larger BDSM community. It seems likely that this embeddedness has both positive and negative impacts. Couples can receive support, learn skills, and gain acceptance in this larger community. But couples might also emulate the exemplars they find in the community rather than developing their own dynamics and protocols. It would have been unfortunate, for example, if the couples in the present study who found it useful to go deeper into their power exchange roles to solve relationship problems had learned from the community that this is the "wrong" thing to do.

Finally, with respect to minority stress, many of the participants in the present study told stories of negative stigma, the necessity of concealment, and the challenge of disclosure, supporting Hammack et al.'s (2019) fourth element.

In sum, the present data generally support Hammack et al.'s (2019) framework for understanding BDSM relationships, differing primarily in a power exchange that seems deeper and more fundamental than "recreational."

\section{Implications for Non-BDSM Relationships}

The present couples clearly communicated their wants and needs, which promoted better relationships and better sex. Western culture, with its championing of rugged individualism and the maximizing of personal gain, seems at odds with intimacy. In this individualist model, it is 
not always prudent to disclose. The interviewed cohorts recognized that by maximizing the gains of their partner, they too got more of what they wanted. We believe these practices and attitudes could be of benefit to all couples regardless of their level of kink.

Jozifkova and Kolackova (2017) found that vanilla relationships with some level of hierarchy (regardless of the dominant partner's gender) had more children, with almost half of respondents finding hierarchical disparity to be sexually arousing. In personal communications (March 19, 2019), Jozifkova noted that when the disparity was excessive, the relationships ended. Given that Dominants in the present study were hesitant and the submissives wanted more, yet both were happy, perhaps the present respondents found the sweet spot of disparity.

\section{Limitations and Future Directions}

Our sample consisted of BDSM couples who were active members of the Arizona Power Exchange. Although a sample size of 33 is sufficient for the qualitative aspects of the present study, it is somewhat low for the quantitative aspects. Further research, ideally with larger samples drawn from other BDSM populations, would be of value in testing the replicability and generalizability of the results.

All the relationships studied consisted of a cisgender woman involved with a cisgender man. Although anecdotal observations suggest equally successful long-term BDSM relationships among gender expansive individuals and among individuals in same-sex relationships, further research with a greater diversity of participants would be of value.

The studied couples deeply valued clarity and transparency in their relationships. Because of the emotionally sensitive nature of BDSM play, the need for open communication may be higher than for the non-kinky population. It is possible that this requirement for communication would lead to closer, more fulfilling relationships than relationships that do not demand this level of communication, but additional research would be needed to test this idea.

\section{Conclusion}

The present data clearly demonstrate that long-term BDSM relationships exist and can be highly functional. For couples that manifest ongoing power exchange, the dynamic appears to serve purposes beyond sexual satisfaction, including facilitating partner bonding and providing a sense of security. Taken as a whole, the participants in this study presented themselves as in loving and committed romantic relationships. They showed a commitment to a high level of communication, to the ongoing practice of vulnerability and intimacy, and to examining and realizing a power structure that was unique to each couple - commitments that, we suspect, would serve both kink and non-kink couples well.

Alison, L., Santtila, P., Sandnabba, N. K., \& Nordling, N. (2001). Sadomasochisticallyoriented behavior: Diversity in practice and meaning. Archives of Sexual Behavior, 30, 1-12.

\section{References}

Amaro, H. (1995). Love, sex, and power: Considering women's realities in HIV prevention. American Psychologist, 50, 437447. 
Baldwin, G. (1993). Ties that bind: Issues, commentaries, and advice. Los Angeles: Daedalus Publishing.

Bauer, R. (2019). BDSM relationships. In B. L. Simula, J. E. Sumerau, \& A. Miller (Eds.) Expanding the rainbow: Exploring the relationships of bi+, polyamorous, kinky, ace, intersex, and trans people (pp. 135-147). Brill Sense, Leden: Boston.

Bean, W. J. (1994). Leathersex: A guide for the curious outsider and the serious player. Los Angeles: Daedalus Publishing Company.

Berger, P. L. \& Luckmann, T. (1966). The social construction of reality. New York: Penguin Books.

Brame, G. G., Brame, W. D., \& Jacobs, J. (1993). Different loving: An exploration of the world of sexual dominance and submission. New York: Villard Books.

Brown, A., Barker, E. D., \& Rahman, Q. (2020). A systematic scoping review of the prevalence, etiological, psychological, and interpersonal factors associated with BDSM. The Journal of Sex Research, 57, 781-811.

Butts, A. M. (2007). "Signed, sealed, delivered ... I'm yours": Calibrating body ownership through the consensual mastery/slavery dynamic. Sex and Culture, 11, 62-76.

Califia, P. (1993). Sensuous magic: A guide for adventurous couples. New York: Masquerade Books, Inc.

Campbell, D. (2000). The bride wore black leather: ... and he looked fabulous! Emeryville, CA: Greenery Press.

Carlson, D. L., Miller, A. J., Sassler, S., \& Hanson, S. (2014). The gendered division of housework and couples' sexual relationships: A re-examination. Journal of Marriage and Family, 78, 975- 995.

Crane, D. R., Middleton, K. C., \& Bean, R. A. (2000). Establishing criterion scores for the Kansas marital satisfaction scale and the revised dyadic adjustment scale. American Journal of Family Therapy, 28(1), 53-60. doi:10.1080/019261800261815

Dancer, P. L., Kleinplatz, P. J., \& Moser, C. (2006). 24/7 SM slavery. Journal of Homosexuality, 50, 81-101.

De Neef, N., Coppens, V., Huys, W., \& Morrens, M. (2019). Bondage-Discipline, DominanceSubmission and Sadomasochism (BDSM) from an integrative biopsychosocial perspective: a systematic review. Sexual Medicine 7(2), 129144.
Dunbar, N. E. (2004). Dyadic power theory: Constructing a communication-based theory of relational power. The Journal of Family Communication, 4, 235- 248.

Graham, B. C., Butler, S. E., McGraw, R., Cannes, S. M., \& Smith, J. (2015). Member Perspectives on the Role of BDSM Communities. The Journal of Sex Research, 53, 895-909.

Hammack, P. L., Frost, D. M., \& Hughes, S. D. (2019). Queer intimacies: A new paradigm for the study of relationship diversity. The Journal of Sex Research, 56, 556-592.

Hébert, A., \& Weaver, A. (2015). Perks, problems, and the people who play: A qualitative exploration of dominant and submissive BDSM roles. Canadian Journal Of Human Sexuality, 24, 49-62.

Holvoet, L., Huys, W. Coppens, V., Seeuws, J., Goethals, K., \& Morrens, M. (2017). Fifty shades of Belgian gray: The prevalence of BDSM-related fantasies and activities in the general population. The Journal of Sexual Medicine, 14, 1152-1159.

James, E. L. (2012). Fifty shades of grey. New York: Vintage Books.

Joyal, C. C., Cossette, A., \& Lapierre, V. (2015). What exactly is an unusual sexual fantasy? The Journal of Sexual Medicine, 12, 328-340.

Jozifkova, E., \& Kolackova, M. (2017). Sexual arousal by dominance and submission in relation to increased reproductive success in the general population. Neuroendocrinology Letters, 38, 381-387.

Kaldera, R. (Ed.). (2014). Paradigms of power: Styles of Master/slave relationships. Hubbardston, Massachusetts: Alfred Press.

Kimble, L. (2017). BDSM expert weighs in on what fifty shades gets right - and wrong - about the lifestyle. People.

https://people.com/movies/bdsm-expert-on-fiftyshades/

Kravitz, J. (2018). What 'fifty shades' gets right \& wrong about BDSM, according to an expert. Elite Daily. https://www.elitedaily.com/p/whatfifty-shades-gets-right-wrong-about-bdsmaccording-to-expert-8224269

Lee, E. M., Erickson, J. M., \& Sagarin, B. J. (2018, April). The diverse world of consensual power exchange relationships. Paper presented at the Midwestern Psychological Association's annual meeting, Chicago, IL.

Lennon, C. A., Stewart, A. L., \& Ledermann, T. (2012). The role of power in intimate relationships. Journal of Social and Personal Relationships, 30, 95-114. 
Money, J. (1986). Lovemaps: Clinical concepts of sexual/erotic health and pathology, paraphilia, and gender transposition in childhood, adolescence, and maturity. New York: Irvington Publishers, Inc.

Pitagora, D. (2013). Consent vs. coercion: BDSM interactions highlight a fine but immutable line. The New School Psychology Bulletin, 10, 27-36.

Pitagora, D. (2015). Intimate partner violence in sadomasochistic relationships. Sexual and Relationship Therapy, 31, 95-108.

Pomeroy, W. B., Flax, C. C., Wheeler, C. C. (1982). Taking a sex history: Interviewing and recording. New York: Macmillan Publishing Co., Inc.

Rogak, H. M., \& Connor, J. J. (2018). Practice of consensual BDSM and relationship satisfaction. Sexual and Relationship Therapy, 33, 454-469.

Rosenfeld, M. J., Thomas, R. J., \& Hausen, S. (2019). Disintermediating your friends: How online dating in the United States displaces other ways of meeting. Proceedings of the National Academy of Sciences, 116, 17753-17758.
Sagarin, B. J., Cutler, B., Cutler, N., Lawler-Sagarin, K. A., \& Matuszewich, L. (2009). Hormonal changes and couple bonding in consensual sadomasochistic activity. Archives of Sexual Behavior, 28, 186-200.

Shahbaz, C., \& Chirinos, P. (2016). Becoming a kink aware therapist. New York: Routledge.

Smith, A. (2015). Fifty shades of grey: What BDSM enthusiasts think. The Guardian. https://www.theguardian.com/film/2015/feb/15/f ifty-shades-of-grey-bdsm-enthusiasts

Townsend, L. (1972). The leatherman's handbook. New York: Olympia Press.

Wiseman, J. (1998). SM 101: A realistic introduction (2nd ed.). Gardena, CA: Greenery Press.

Wismeijer, A. A. J., \& van Assen, M. A. L. M. (2013). Psychological characteristics of BDSM practitioners. Journal of Sexual Medicine, 10, 1943-1952. 